\title{
55. Calcium Dependent Receptor Potential of the Electroreceptor of Marine Catfish
}

\author{
By Yoshiko AKutsu and Shosaku OBara \\ Department of Physiology, Teikyo Univ., School of Med., \\ Kaga 2-11-1, Itabashi-ku, Tokyo 173 \\ (Comm. by Yasuji Katsuki, M. J. A., March 12, 1974)
}

Receptor potential in the secondary sensory receptor is defined as an initial electrical response following sensory input, which is an intermediate before transmitting synaptically to afferent nerves. Because of the role of receptor cell in the causal sequence in sensory information processing, the receptor potential can be regarded as a presynaptic potential, as well as the stimulus-dependent response. Ionic mechanism of the receptor potential, therefore, should be of general importance.

Electroreceptors are modified acoustico-lateralis receptors, and in some of them ${ }^{1,2)}$ receptor potentials are clearly discernible from electrotonic potential (stimulus induced component). In the previous report, ${ }^{3)}$ ampullae of Lorenzini of marine catfish, Plotosus anguillaris (Lacépède), has been shown to be a tonic electroreceptor. Their sensory epithelium forms a nearly spherical ampulla (Fig. 1) which is connected through a long transparent duct to distant skin surface and to open to exterior. On anodal stimulation, the ampulla in situ shows graded or quasi-regenerative positive-going responses (RP), which are facilitatory in terms of the generator potential and afferent discharge. ${ }^{3)}$ This RP is a mass response of the sensory epithelium, and presumably originates as a depolarizing activity at the innervated surface of receptor cells which faces the extra-ampulla medium. It is expected, therefore, that the RP should be affected by variations in ionic composition in the extra-ampulla medium. In the present report these hypothesis were tested, and Ca-dependency of $\mathrm{RP}$ was confirmed by a simple method.

The experiments were performed on ampullae of Lorenzini, which were isolated together with their ducts from curarized fish. The duct was introduced into a suction glass capillary by the cut end, and suspended over the fluid surface with only the ampulla remaining in flowing saline, which could be changed successively to test solutions. The glass capillary was equipped with two $\mathrm{Ag}-\mathrm{AgCl}$ wires, one with large series resistor for electrical stimulation and the other insulated except at its tip for recording through a high 
A

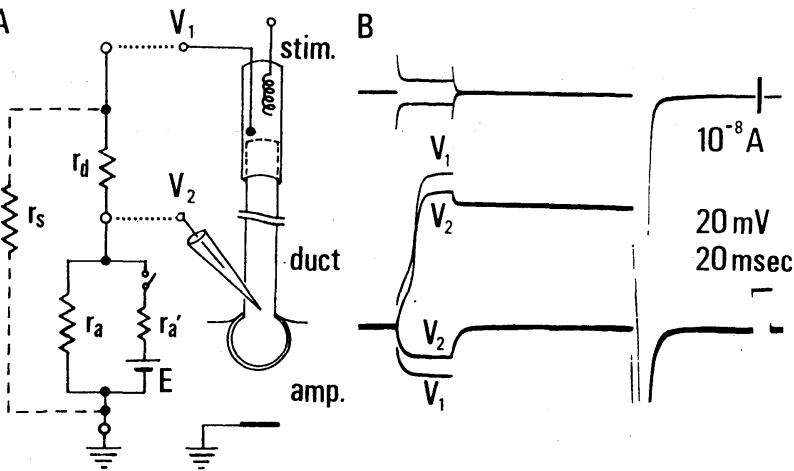

Fig. 1. Responses of the ampulla recorded at two points. A: right; arrangement for stimulation and recording. amp: the ampulla. duct: the long duct $(1.5-2 \mathrm{~cm})$, cut end of which was placed in suction electrode. stim: stimulating electrode. $V_{1}$ and $V_{2}$ : responses recorded in the duct and near the ampulla. left: equivalent circuit. $r_{s}$ : leakage resistance along the duct. $r_{d}$ : longitudinal resistance through the duct. $r_{a}$ : effective resistance of the ampulla at rest. $\mathrm{E}$ : electromotive force of RP. $\mathrm{ra}^{\prime}$ : resistance in series to $\mathrm{E}$. RP is generated by closing the switch. Capacitative components are omitted for simplicity. B: Sample record of the ampulla. upper trace: stimulating current, negative pulse downward. lower trace: superimposed records at two recording sites.

input impedance preamplifier. A large indifferent electrode of $3 \mathrm{M}$ $\mathrm{KCl}$ agar grounded the bathing medium (see Fig. 1A).

That with this simple arrangement the RP in the ampulla could be recorded unattenuated in the duct was demonstrated in Fig. 1. With an isolated ampulla prepared as before, an additional recording was made by penetrating the duct close to ampulla with a glass microelectrode $\left(\mathrm{V}_{2}\right)$, and was compared with that of the suction capillary $\left(V_{1}\right)$. Electrical stimuli with cathode to the capillary produced linear negative deflections with exponential rise and fall, $\mathrm{V}_{1}$ being larger than $V_{2}$. Stronger anodal stimuli could evoke a quasiregenerative response at certain voltage threshold, that was maintained after the stimulating current was terminated. The regenerative response thus evokedw as identical to the RP observed in in situ preparation, because the afferent nerve discharge, when recorded simultaneously, showed a strong facilitation during the response. Presumably strong anodal stimuli activated the whole sensory epithelium simultaneously so that the response became sufficiently regenerative. Hence the response will be called a regenerative receptor potential (RP).

Noteworthy was that $V_{1}$ and $V_{2}$ became identical for the regenerative RP after allowing a proper desiccation along the duct. A 
simplified equivalent circuit was shown also in Fig. 1. When an external leakage $\left(r_{s}\right)$ along the duct was minimized, longitudinal current due to the RP (E) became negligible and $V_{1}$ in the duct could represent the $\mathrm{RP}$ in the ampulla. In another words, the ampulla could be electrically isolated from the rest of the structure.

For the later experiments, therefore, only the suction electrode arrangement was used to observe the regenerative RP. The negative responses on a given cathodal pulse were also monitored intermittently to assess a contribution of $r_{s}$, when the external medium was changed. An additional strong cathodal pulse was given to abolish the maintained RP (Fig. 1), and the RP could be evoked repeatedly with little effect of refractoriness. One drawback of this method was that the large conductance increase associated with the RP would be obscured because of the longitudinal resistance $\left(r_{d}\right)$ in series to the active ampulla $\left(r_{a} / / r_{a}{ }^{\prime}\right)$. The conductance increase was confirmed in separate experiments such as shown in Fig. 1. Excessive desiccation resulted in an apparent increase of combined resistances and in a deterioration of $\mathrm{RP}$, so that care was taken to maintain a proper humidity around the preparation.

Control saline was based on analysis of fish serum, and slightly modified: $\mathrm{NaCl} 200 \mathrm{mM}, \mathrm{KCl} 5 \mathrm{mM}$ and $\mathrm{CaCl}_{2} 3 \mathrm{mM}$; $\mathrm{pH}$ was adjusted to 7.2-7.4 with Tris buffer. High Ca saline was prepared by replacing a part of $\mathrm{NaCl}$ with $\mathrm{CaCl}_{2}$ isosmotically. When $\mathrm{Na}$ or $\mathrm{K}$ was changed, $\mathrm{NaCl}$ was replaced by osmotically equivalent amount of sucrose, Tris or choline, and $\mathrm{KCl}$ was substituted by $\mathrm{NaCl}$. Tetrodotoxin (TTX), $\mathrm{MnCl}_{2}$ or $\mathrm{MgCl}_{2}$, when used, was added directly to the saline. All experiments were performed at room temperature $\left(18-24^{\circ} \mathrm{C}\right)$.

Change from control saline to high Ca saline was regularly followed by an reversible increase of the RP amplitude, while DC potential of the ampulla was little changed. The $\mathrm{RP}$ reached the new steady state about 3 minutes after the exchange of solution. The RP amplitude was linearly proportional to the logarithm of Ca concentration for the range of 1-30 mM with a slope of c. $23 \mathrm{mV}$ for a tenfold change (Fig. 2), which was a fairly good approximation of the theoretical value expected from the Nernst equation $(29 \mathrm{mV})$. Approximate voltage threshold also increased with elevated Ca concentration. Outside of the range, however, it became in general difficult to evoke the RP. Changes in Na concentration had little effect on the RP, which persisted after total replacement with Tris, choline or sucrose long after the time when the RP should be affected by changes in Ca. The Na-free saline, however, often lead reversibly to a deterioration of the RP following small spontaneous depolarization for unknown 


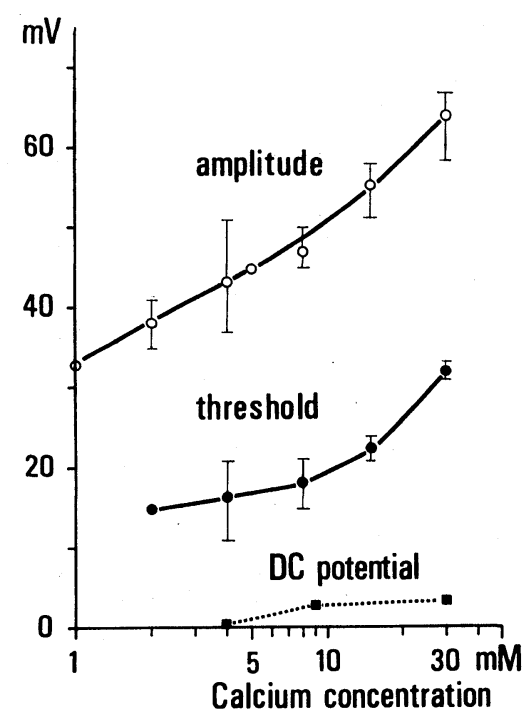

Fig. 2. The effects of $\mathrm{Ca}$ on the RP. Open circles: mean values of amplitude of the regenerative RP (more than 6 measurements for each). Filled circles: approximate voltage threshold. Vertical bars indicate the range of variations at respective $\mathrm{Ca}$ concentration. Filled square: DC potentials of the ampulla.

reasons. Changes in $\mathrm{K}$ concentration $(5-100 \mathrm{mM})$ gave no effect on the RP and DC potential of the ampulla. The Ca-dependency of the $\mathrm{RP}$ was further tested as follows. The regenerative RP was not affected by TTX of $10^{-6} \mathrm{~g} / \mathrm{ml}$ which should be more than 100 times the effective amount for suppression of the Na-spikes, while afferent nerve discharges were irreversibly blocked. Effects of $\mathrm{Mn}$ and $\mathrm{Mg}$ ions, which were proved to inhibit the initiation of Ca-spikes, ${ }^{4)}$ was explored at the concentration of 5 and $10 \mathrm{mM}$ in the presence of $5 \mathrm{mM}$ Ca. The regenerative $\mathrm{RP}$ was competitively blocked by $10 \mathrm{mM} \mathrm{Mn}$, while at $5 \mathrm{mM}$ no significant change was found. The RP was also suppressed by $10 \mathrm{mM} \mathrm{Mg}$ but the effect was weaker than $\mathrm{Mn}$. On return to control saline the $\mathrm{RP}$ reappeared rapidly, though full recovery seldom occured. The effective resistance of the ampulla appeared to change very little for all these cases.

These results confirm first the proposed site for the RP at the innervated surface of the receptor cell, and also provide a direct evidence that the RP in the tonic electroreceptor is due to the conductance increase toward Ca. In phasic electroreceptors of the fresh water teleost, the receptor activity has been shown to occur at the innervated face, which is reportedly not affected by TTX, ${ }^{5)}$ suggesting its Ca-dependency. In another sensory receptors, the Ca-depend- 
ent response has been verified in Paramecium in response to mechanical stimuli. ${ }^{6}$ ) Since the RP in the present case serves as a presynaptic potentials as already suggested, the observed Ca-dependent activity of the innervated membrane may play an important role for the transmitter release at the receptor-afferent synapses. The Ca influx at the presynaptic nerve terminal has been implicated in the release of neurotransmitter substance. ${ }^{7)}$

Acknowledgement. The authors are grateful to Mito Aquarium, Numazu-city, Shizuoka, for the generous and continuing supply of the fish. The ionic composition of fish serum was measured by Mr. S. Umekita. This work was supported in part by the grant 837005 from the Ministry of Education of Japan.

\section{References}

1) Bennett, M. V. L. (1972): Fish Physiology, V, 493.

2) Obara, S., and M. V. L. Bennett (1972): J. Gen. Physiol., 60, 534.

3) Obara, S., and Y. Oomura (1973): Proc. Japan Acad., 49, 213.

4) Hagiwara, S., and S. Nakajima (1966): J. Gen. Physiol., 49, 793.

5) Zipser, B., and M. V. L. Bennett (1973): Brain Research, 62, 253.

6) Naitoh, Y., and R. Eckert (1969): Science, 164, 963.

7) Katz, B., and R. Miledi (1969) : J. Physiol., 203, 459. 